\title{
Deduction of skin friction by Clauser technique in unsteady turbulent boundary layers
}

\section{G. J. Brereton}

Dept. of Mechanical Engineering and Applied Mechanics, The University of Michigan, Ann Arbor, MI 48109, USA

\section{Introduction}

In studies of unsteady (oscillatory) turbulent boundary layers, the mean friction velocity is a quantity useful both for evaluating drag coefficients and normalizing experimental data. While it may be measured indirectly using scalartransport probes - hot-film gauges or electrochemical probes - these devices are known to be problematic when fluid flow and scalar transport are not quasi-steady, or if fluid flow is not unidirectional. The notion of deducing $u_{\tau}$ by the Clauser technique - force-fitting a mean-velocity function over part of a profile of $U$ - has obvious attractions, though no justification exists for its extension to mean profiles of unsteady flows. Using results from a recent study of unsteady turbulent boundary layers (Brereton and Reynolds 1987), in which $u_{\tau}$ could be gauged from the near-wall velocity gradient and estimated by Clauser technique, the plausibility of extending the Clauser technique to this application is considered.

\section{Background}

When the Couette-flow assumption is valid near the wall in an oscillatory turbulent boundary layer, the mean $x$-momentum equation may be written using the triple decomposition proposed by Hussain and Reynolds (1970):

$\frac{\tau}{\tau_{0}} \approx 1+p^{+} y^{+}$

where

$\tau_{0}=\left.\mu \frac{\mathrm{d} U}{\mathrm{~d} y}\right|_{y=0}$

$p^{+}=\frac{\mu}{\tau_{0}} \sqrt{\frac{\varrho}{\tau_{0}}}\left(-U_{\infty} \frac{\mathrm{d} U_{\infty}}{\mathrm{d} x}-\frac{\partial}{\partial x}\left(\overline{\tilde{u}_{\infty} \tilde{u}_{\infty}}-\overline{\tilde{v}_{\infty} \tilde{v}_{\infty}}\right)\right)$,

and

$\tau=\mu \frac{\mathrm{d} U}{\mathrm{~d} y}-\varrho \overline{u^{\prime} v^{\prime}}-\varrho \overline{\tilde{u} \tilde{v}}$.

It differs from its steady-flow form only through an additional stress due to the oscillatory motions $(-\varrho \overline{\tilde{u} \tilde{v}})$ and a modified pressure-gradient term. If the contributions of $-\varrho \overline{u^{\prime} v^{\prime}}$ and $-\varrho \overline{\tilde{u} \tilde{v}}$ to the shear stress are small very close to the wall (say $y^{+}<7$, where viscous flow dominates), $u_{\tau}$ may be esti- mated from a fit of $U$ against $y$ which follows from integration of (1). The Clauser technique could be applied dependably (in the form $u^{+}=\frac{1}{x} \ln y^{+}+C$ ) over part of a profile of $U$ where $\tau$ were approximately constant and $-\overline{u^{\prime} v^{\prime}}-\overline{\tilde{u} \tilde{v}}$ scaled upon $\mathrm{d} U / \mathrm{d} y$ and the local length scale $(y)$ through $\chi$, the Von Kármán constant for steady flow.

\section{Experimental procedure}

In the experiments of this study, a sinusoidal motion of prescribed amplitude was superimposed on an otherwise steady mainstream beyond a turbulent boundary layer $\left(R e_{\theta} \simeq 3200\right)$. The mean pressure gradient was adverse and the amplitude of the oscillatory motion in the free stream increased with downstream distance (at the rate at which $U_{\infty}$ decreased). Thus $\partial \tilde{u}_{\infty} / \partial x \neq 0$ and $\overline{\tilde{u} \tilde{v}}$ was not necessarily zero. For a given amplitude of $\tilde{u}_{\infty}$, its frequency could be varied between a lower limit (quasi-steady flow) and an upper one at which Stokes' solution was matched. A series of experiments was conducted for oscillation at $0.2,0.5,0.8$, $1.0,1.6$ and $2.0 \mathrm{~Hz}$, with mean boundary conditions of $\beta=3.2\left(\right.$ where $\beta$ is the Clauser parameter, $\left.\frac{\delta^{*}}{\tau_{0}} \frac{\mathrm{d} P_{\infty}}{\mathrm{d} x}\right)$ and the amplitude of $\tilde{u}_{\infty}$ equal to $11 \%$ of $U_{\infty}$.

Measurements of the boundary-layer velocity field were made with a two-colour laser-Doppler anemometer operated in forward-scatter mode, in a closed-loop water tunnel. The lengths and diameters of the measuring volumes were about $0.5 \mathrm{~mm}$ and $0.15 \mathrm{~mm}$ respectively within a boundary layer for which $\delta_{99} \simeq 80 \mathrm{~mm}$.

\section{Results and discussion}

The measurement techniques were applied first in steady turbulent boundary layers with $R e_{\theta} \simeq 3200$, at eight different pressure gradients in the range $0 \leq \beta \leq 6$, as a check upon their relative accuracies. A least-squares fit of $u^{+}=y^{+}$(for $y^{+}<7$, typically four or more data points) was used to evaluate $u_{\tau}$ for each case. The Clauser technique was applied to each profile as a least-squares fit of Coles' mean velocity function (Coles 1968), in which $u_{\tau}$ and $\delta$ were varied systematically. As this function also represented the wake, it could 


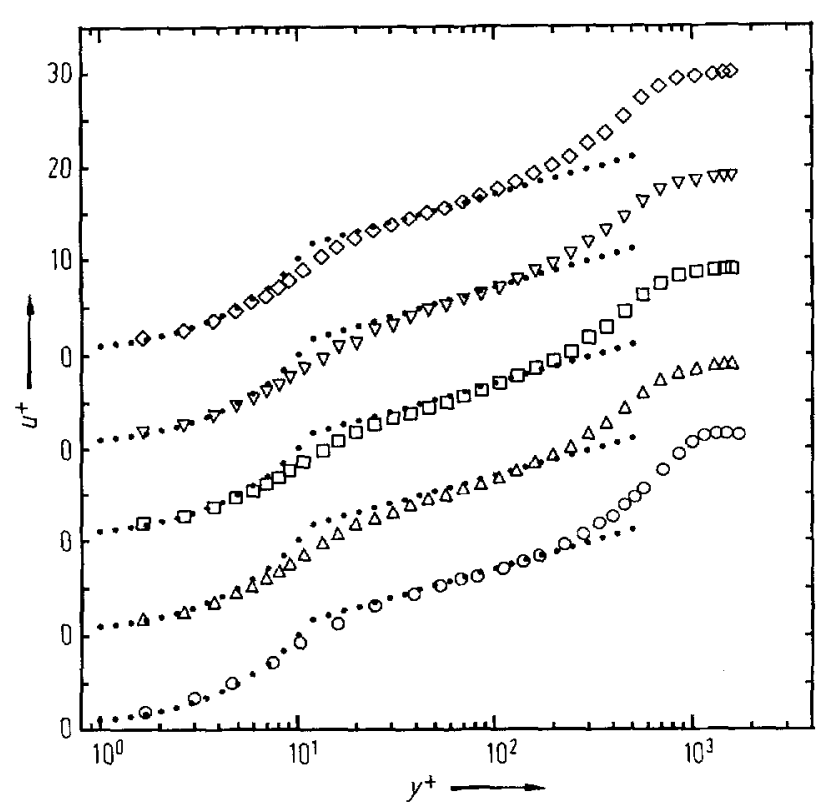

Fig. 1. Boundary-layer profiles of $U$ in wall units, with fitted functions; $\bullet u^{+}=y^{+}, u^{+}=\frac{1}{0.4} \ln y^{+}+5.5 ;$ steady flow; $\triangle 0.2 \mathrm{~Hz}$; $\square 0.5 \mathrm{~Hz} ; \nabla 1.0 \mathrm{~Hz} ; \diamond 2.0 \mathrm{~Hz}$; in all cases $\beta=3.2$

be applied beyond the outermost limits of the log-linear region and so was used over the recommended range of $50<y^{+}$and $y / \delta<0.75$ (at least 10 data points). When $x$ and $C$ were taken as 0.4 and 5.5 respectively in the function $u^{+}=\frac{1}{x} \ln y^{+}+C+\frac{2 \Pi}{x} \sin ^{2}\left(\frac{\pi}{2} \frac{y}{\delta}\right)$, excellent fits resulted with r.m.s. scatter less than $0.2 \%$. The values of $u_{\tau}$ deduced by each technique compared very favourably over the entire range of $\beta$, with discrepancies no larger than $2 \%$. Thus near-wall measurements of $U$ were considered accurate and concerns over increased experimental uncertainty in this region appeared unwarranted. To emphasize the significance of such low discrepancies, the effect of replacing the values of $x$ and $C$ of 0.4 and 5.5 with 0.41 and 5.0 was to increase $u_{\tau}$ by $4 \%$ in each case!

For time-averaged unsteady flow $(\beta=3.2)$, measured profiles of $U$ are shown in Fig. 1 at several representative frequencies of oscillation, together with a steady profile at the same value of $\beta$. The linear and $\log$-linear parts of the forced fits are superimposed, for $\chi=0.4$ and $C=5.5$. It is evident that the fits to unsteady profiles are poor, and that smaller. values of both $x$ and $C$ are appropriate. In fact, for systematic variation of $u_{\tau}, \delta, \varkappa$ and $C$, the closest fits to these data were obtained with $x \simeq 0.33$ and $C \simeq 3.0$, with only slight modifications to $u_{\tau}$. Nonetheless the values of $u_{\tau}$ deduced from these fits differed from their near-wall counterparts by only $3 \%-4 \%$ - a surprisingly good result in view of the poorness of the fit.

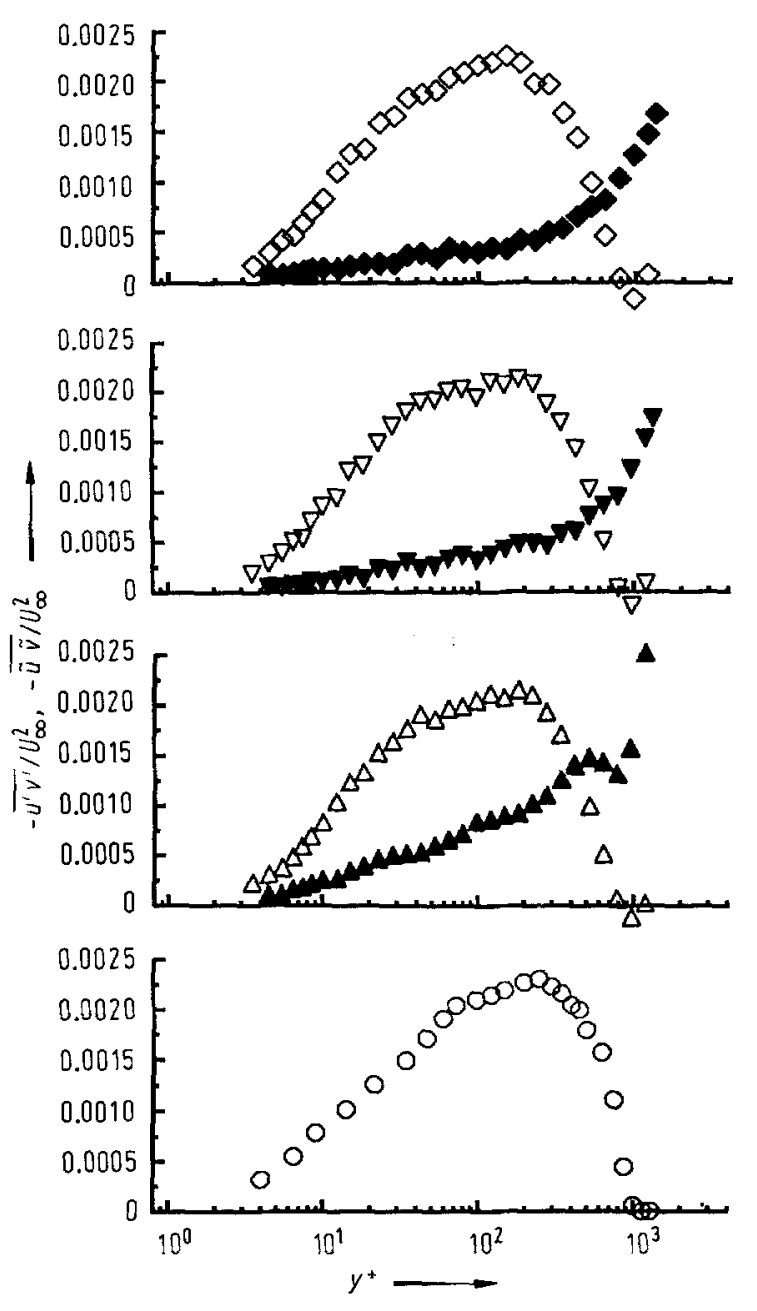

Fig. 2. Turbulent and oscillatory stresses; symbols describing $-u^{\prime} v^{\prime}:$ o steady flow; $\triangle 0.2 \mathrm{~Hz} ; \nabla 1.0 \mathrm{~Hz} ; \diamond 2.0 \mathrm{~Hz}$; filled symbols denote the corresponding measures of $-\tilde{u} \tilde{v}$; in all cases $\beta=3.2$

To explain these findings more clearly, profiles of $-\overline{u^{\prime} v^{\prime}}$ and $-\overline{\tilde{u} \tilde{v}}$ are shown in Fig. 2. A reference profile of $-\overline{u^{\prime} v^{\prime}}$ for steady flow at $\beta=3.2$ is also included. This profile is similar in both shape and magnitude to the unsteady-flow profiles, which are characterized by a region of approximately constant stress $\left(-\overline{u^{\prime} v^{\prime}}-\overline{\tilde{u} \tilde{v}} \simeq 0.0025 U_{\infty}^{2}\right)$ over the range $30<y^{+}<200$, the log-linear region of Fig. 1. Although $-\tilde{\tilde{u}} \tilde{v}$ is small within this region, it still reaches between $10 \%$ and $20 \%$ of $-\overline{u^{\prime} v^{\prime}}$, with larger values at lower frequencies.

As $\varkappa$ (in steady flow) relates a time-dependent velocity scale $\sqrt{-\overline{u^{\prime} v^{\prime}}}$ to a local mean one $\mathrm{d} U / \mathrm{d} y$ (via $y$, the only relevant length scale), the suitability of a smaller $\varkappa$ for this oscillatory flow indicates that any augmentation by $-\overline{\tilde{u}} \tilde{v}$ of the velocity scale is less important than its local effect in increasing $\mathrm{d} U / \mathrm{d} y$. Consequently accurate values of $u_{\tau}$ (within $4 \%$ ) generated by an ill-fitting application of the Clauser technique appear to be the result of a fortuitous choice of 
fitting range. Therefore, without prior knowledge of unsteady-flow values of $x$ and $C$, this technique should be applied only with great caution to unsteady turbulent boundary layers in which the stress $-\varrho \overline{\tilde{u}} \tilde{v}$ is present.

\section{Acknowledgements}

The contributions of Profs. W. C. Reynolds and J. P. Johnston of Stanford University and the support of the US Army Research Office are gratefully acknowledged.

\section{References}

Brereton, G. J.; Reynolds, W. C. 1987: Experimental study of the fluid mechanics of unsteady turbulent boundary layers. Rep. TF-29, Dept. Mech. Eng., Stanford University, Stanford/CA

Coles, D. E. 1968: The young person's guide to the data. In: Proc. comput. turbulent boundary layers - AFOSR-IFP-Stanford Conference. (eds. Coles, D. E.; Hirst, E. A.). Vol. 2, pp. 1-46. Dept. Mech. Eng., Stanford University, Stanford/CA

Hussain, A. K. M. F.; Reynolds, W. C. 1970: The mechanics of an organized wave in turbulent shear flow. J. Fluid Mech. 41, $241-261$

Received September 27, 1988

\title{
Spatial resolution and downwash velocity corrections for multiple-hole pressure probes in complex flows
}

\author{
P. M. Ligrani \\ Dept. of Mechanical Engineering, Code 69Li, Naval Postgraduate School, Monterey, CA 93943-5000, USA

\section{B. A. Singer} \\ High Technology Corp., 28 Research Drive, Hampton, VA 23666, USA
}

\section{R. Baun}

Dept. of Mechanical Engineering, Naval Postgraduate School, Monterey, CA 93943-5000, USA

\section{Introduction}

Methods to account for finite spatial resolution and induced downwash velocity are given for multiple-hole pressure probes as they are used to measure complex three-dimensional flow fields. Spatial resolution limitations result because pressures from different ports are not measured at the same physical location. As transverse gradients increase in magnitude, uncorrected errors then become larger. Because of this, most existing correction techniques employ schemes which depend on gradients of velocity or pressure (Ikui and Inoue 1970; Sitaram et al. 1981; Eibeck and Eaton 1985; Westphal et al. 1987). A simpler and more sensible approach for a five-hole probe corrects pressures so that all appear to be measured at the location of the central hole by accounting for the exact spacing between different pressure ports.

Induced downwash velocity is part of the secondary flow which occurs as a result of the blockage of blunt bodies within transverse gradients of mean streamwise velocity. Young and Mass (1936) studied this phenomena as it results from flat faced pitot probes, showing that measured boundary layer velocities correspond to positions farther from the wall than the probe position. Livesey (1956) extended these results to demonstrate that apparent streamline displacement depends strongly on probe shape. In the present technical note, induced down- wash velocity is corrected by assuming it to be proportional to transverse gradients of streamwise velocity.

\section{Experimental apparatus and procedures}

Two five hole angle-type pressure probes are used in this study. One of these is a United Sensor DC-250-24CD conical five-hole probe with a tip diameter of $6.35 \mathrm{~mm}$. The other is a miniature five-hole probe with a $1.22 \mathrm{~mm}$ tip diameter. The probes are calibrated in the uniform freestream of an open circuit blower tunnel located in the laboratories of the Department of Mechanical Engineering at the Naval Postgraduate School. Celesco model .LCVR variable reluctance transducers with a full scale pressure range of $2 \mathrm{~cm}$ of water are used with the United Sensor probe. Validyne model DP 103-06 variable reluctance transducers with a full scale range of $0.25 \mathrm{~cm}$ of water differential pressure are used with the miniature probe. Calibration and measurement procedures to obtain timeaveraged measurements are described in detail by Ligrani et al. (1988) for the smaller probe, and by Williams (1988) for the larger probe.

\section{Spatial resolution correction}

Figure 1 illustrates how the present spatial resolution scheme is applied. Here, the $x$ coordinate represents the 